\title{
Efficient Encoding of Matrix Microparticles with Nanocrystals for Fluorescent Polyelectrolyte Microcapsules Development
}

\author{
Nifontova G.0. ${ }^{1}$, Sukhanova A.V. ${ }^{1,2}$, Samokhvalov P.S. ${ }^{1}$, and Nabiev I.R. ${ }^{1,2}$ \\ ${ }^{1}$ National Research Nuclear University MEPhl (Moscow Engineering Physics Institute), \\ Kashirskoe shosse 31, Moscow, 115409, Russia \\ ${ }^{2}$ Laboratoire de Recherche en Nanosciences, EA4682-LRN, Université de Reims Champagne- \\ Ardenne, Reims, France
}

\section{Abstract}

Polyelectrolyte microcapsules development and further use as specific carriers for drug molecules, fluorescent dyes, and metal nanoparticles is a promising approach to designing theranostic agents. Semiconductor nanocrystal quantum dots exhibiting size-dependent optical properties, a high photostability, and optimal fluorescent

Corresponding Author:

Nabiev I.R.

igor.nabiev@gmail.com

Received: 17 January 2018

Accepted: 25 March 2018

Published: 17 April 2018

Publishing services provided by Knowledge E

(c) Nifontova G.O. et al. This article is distributed under the terms of the

Attribution License, which permits unrestricted use and redistribution provided that the original author and source are credited.

Selection and Peer-review under the responsibility of the PhysBioSymp17 Conference Committee.

\section{G OPEN ACCESS} properties can be advantageous over classical organic fluorophores. The results of elaboration of efficient encoding of matrix microparticles with nanocrystals for development of fluorescent polyelectrolyte microcapsules and the characteristics of the obtained encoded microbeads are demonstrated.

Keywords: Semiconductor nanocrystals; encoding of matrix microbeads; theranostic agents, polyelectrolyte microcapsules, layer-by-layer technique.

\section{Introduction}

Development of theranostic agents serving simultaneously as therapeutically active agents and imaging tools for early diagnosis of various diseases is an important task in designing the systems for controlled drug delivery $[1,2]$. Engineering of polyelectrolyte microcapsules which represent supramolecular assemblies of polyelectrolyte complexes is an efficient approach to combining both functions.

The existing technologies of microcapsule fabrication allow biologically active compounds and metal, magnetic, or fluorescent semiconductor nanoparticles to be incorporated into the capsules or tag them [3]. The layer-by-layer technique is the most effective way of polyelectrolyte microcapsule preparation, with oppositely charged polymers, a polyanion and a polycation, successively adsorbed on the microbead surface, which are generally used as templates. Biocompatible polymers, such as chitosan, protamine dextran, poly-I-lysine, poly-I-arginine, dextrane sulfate, poly(sodium 
styrene sulfonate), and poly(allylamine), are widely used in polyelectrolyte microcapsule formation [4].

Quantum dots (QDs) are fluorescent semiconductor nanocrystals characterized by a high photostability, wide absorption spectrum, and narrow and symmetrical fluorescence spectrum with the peak position determined by the size of the nanoparticle, as shown in Fig. 1. Owing to the optical characteristics of QDs, a single source of radiation can be used to excite QDs of different fluorescent colors, which makes them promising fluorophores for multiplexed imaging $[5,6]$.

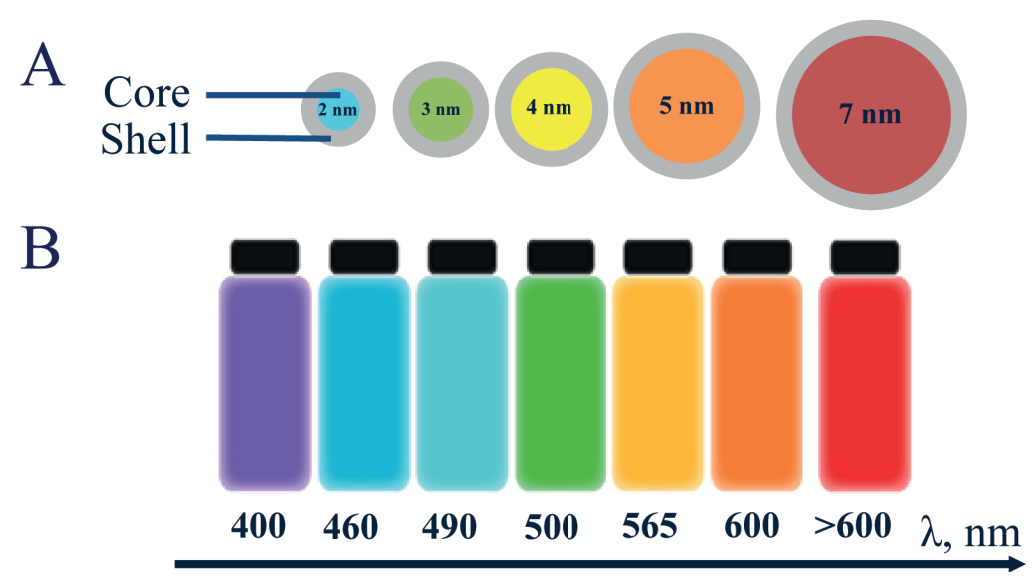

Figure 1: The structure of the QD (A) and the dependence of QD fluorescence properties on their size (B).

Here, we have developed the technology for encoding of template matrix microparticles with semiconductor nanocrystals using a modified method of alternate layer-bylayer application of oppositely charged polymers and water-soluble QDS.

\section{Materials and methods}

\subsection{QD solubilization and characterization}

CdSe/ZnS core/shell QDs were synthesized and kindly provided by Dr Pavel Samokhvalov (Laboratory of Nano-Bioengineering, Moscow Engineering Physics Institute, Moscow, Russia). Originally water-insoluble CdSe/ZnS QDs were solubilized with carboxylated polyethylene glycol derivatives as described earlier [6].

The hydrodynamic diameter and $\zeta$-potential of water-soluble QD were determined by dynamic light scattering and laser Doppler micro-electrophoresis, respectively, using a Zetasizer NanoZS analyzer (Malvern, UK). 


\subsection{Microbead encoding with QDs}

Home-made calcium carbonate microspherolites were synthesized by the precipitation technique [7] and used as template cores in microbeads preparation. The polycation poly(allylamine hydrochloride) (PAH, Sigma-Aldrich, USA) and the polyanion (sodium 4-styrensulfonate) (PSS, Sigma-Aldrich, USA), were used for polyelectrolyte layersformation.

Encoding of microbeads with QDs was performed using the modified method of layer-by-layer deposition of alternately charged polymer layers and water-soluble QDs [8]. Calcium carbonate micropartilces were suspended in ultrapure water; then, the polyelectrolyte aqueous solution containing sodium chloride was added, and the obtained suspension was sonicated and incubated while shaking. Afterwards, the excess of polymer was removed by successive washings and resuspendings in ultrapure water. Negatively charged water-soluble QDs were adsorbed at the polycationcovered microbead surface and further covered by the additional alternately charged polyelectrolyte layers. Microbeads were encoded with different amounts of QDs (from 0.3 to $2 \mathrm{mg}$ of QDs). The resultant encoded microbeads were stored at $4^{\circ} \mathrm{C}$ in the dark.

\subsection{Encoding efficiency determination}

The efficiency of the encoding was estimated spectrophotometrically. The QD content in the supernatant after their adsorption at the microbeads surface and centrifugation was evaluated, and the amount of the absorbed QDs $\left(\mathrm{Q}_{Q D \text { sabs }}\right)$ was calculated using the following equation: $\mathrm{Q}_{Q D \text { sabs }}=\mathrm{Q}_{Q D \text { sin }}-\mathrm{Q}_{Q D \text { ss }}$ where $\mathrm{Q}_{Q D \text { sin }}$ is the initial content of QDs for microbeads encoding and $Q_{Q D s s}$ is the QD content in the supernatant.

\subsection{Fluorescence microscopy}

The morphology and size distribution of the microbeads were analyzed using Carl Zeiss Axio Scope A1 microscope (Carl Zeiss, Germany). The QD-encoded microbeads samples for the microscopy study contained $20 \%$ glycerol and were mounted at SuperFrost ${ }^{T M}$ microscope slides. Imaging of the probes was performed using Texas Red optical filter (ex $596 \mathrm{~nm} / \mathrm{em} 615 \mathrm{~nm}$ ), image processing was performed using the ZEN software (Carl Zeiss, Germany). 


\section{Results}

The water-soluble QDs were characterized by a narrow size distribution from 10 to $12 \mathrm{~nm}$ (Fig. 2) and a negative surface charge (-21.9 $\pm 0.4 \mathrm{mV})$, which ensured their efficient adsorption between polyelectrolyte layers during the process of encoding.

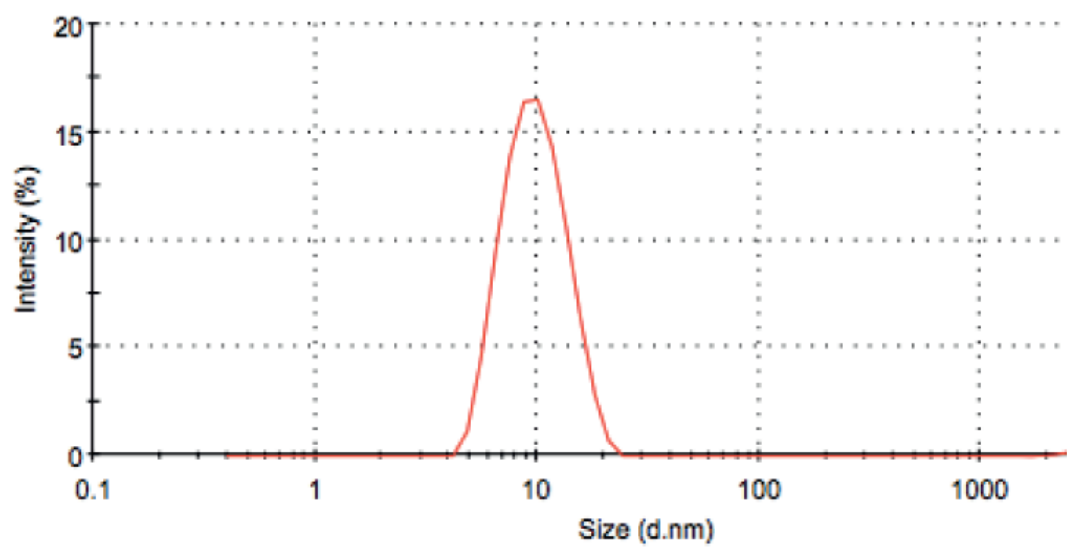

Figure 2: Size distribution of water-soluble CdSe/ZnS core/shell QDs.

The obtained calcium carbonate microbeads were found to have a slightly negative surface charge $(-5.4 \pm 2.5 \mathrm{mV})$, which allows subsequent polyelectrolyte layer growth to be carried out. The resultant microbeads had ten polyelectrolyte layers deposited. Evaluation of the encoding efficiency has demonstrated the amount of QDs embedded on microbeads surface to be $4.8 \times 10^{-5} \mu$ g per microbead.

Fluorescence microscopy data have demonstrated that the encoding procedure used provides the QD content in the microbeads sufficient for contrast imaging, as seen in Fig. 3. Microscopy study has also shown that the size of the developed QD-encoded microbeads vary within a narrow range, from 3 to $6 \mu \mathrm{m}$.

\section{Discussion}

Thus, the layer-by-layer deposition is a promising technique to encapsulate different agents, including QDs. Encoding of microbeads with QDs is based on successively applying alternately charged polyelectrolyte layers of polycation PAH and polyanion PSS and solubilized QDs. Interaction between positively charged primary amino groups of PAH and negatively charged sulfo groups of PSS results in interpolymer complex formation and polymeric coating of calcium carbonate microbeads. Carboxylic functional groups of solubilized QDs promote their electrostatically induced adsorption on the interpolymer complex surface where the upper layer is positively charged. Subsequent 


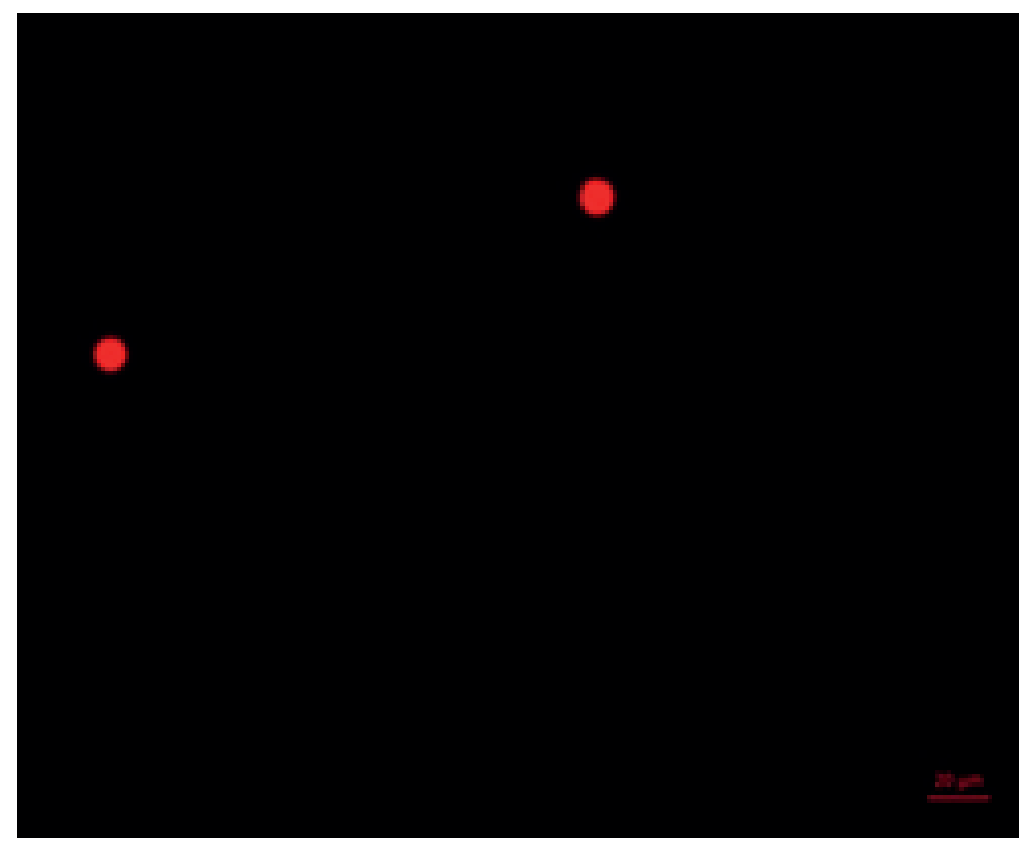

Figure 3: A typical fluorescence microscopy image of microbeads encoded with water-soluble CdSe/ZnS core/shell QDs.

polyelectrolyte layer deposition onto QDs leads to their incorporated in the polymeric coat structure.

Control of the imaging properties of the obtained QD-encoded microbeads is one of the critical points of the evaluation of the developed encoding technique efficiency, which indicates the possibility of their use in microcapsule preparation. The developed microbeads provide contrast imaging in vitro and are distinguished as individual colored objects.

\section{Conclusion}

The data show that the obtained QD-encoded microbeads are characterized by an optimal dispersity and bright fluorescence, which demonstrates the efficiency of the developed procedure of microbead encoding and paves the way to the development of fluorescent polyelectrolyte microcapsules on their basis.

\section{Acknowledgments}

This study was supported by the Ministry of Education and Science of the Russian Federation, State Contract no. 16.1034.2017/ПЧ. 


\section{References}

[1] Xiong R., Soenen S.J., Braeckmans K., Skirtach A.G. (2013) Towards theranostic multicompartment microcapsules: in-situ diagnostics and laser-induced treatment, Theranostics, 3, 141-151. DOI: 10.7150/thno.5846.

[2] Yu W., Chen Y., and Mao Zh. (2016) Hollow Polyelectrolyte Microcapsules as Advanced Drug Delivery Carriers, Journal of Nanoscience and Nanotechnology, 16, 5435-5446. DOI: 10.1166/jnn.2016.11748.

[3] De Geest B.G., De Koker S., Sukhorukov G.B., Kreft O., Parak W.J., Skirtach A.G. , Demeester J., De Smedt S.C., Hennink W.E. (2009) Polyelectrolyte microcapsules for biomedical applications, Soft Matter, 5, 282-291. DOI: 10.1039/B808262F.

[4] Zhao Q., Li B. (2008) pH-controlled drug loading and release from biodegradable microcapsules, Nonomedicine: NBM, 4, 302-310. DOI: 10.1016/j.nano.2008.06.004.

[5] Bilan R., Ametzazurra A., Brazhnik K., Escorza S., Fernández D., Uríbarri M., Nabiev I., Sukhanova A. (2017) Quantum-dot-based suspension microarray for multiplex detection of lung cancer markers: preclinical validation and comparison with the Luminex xMAP® ${ }^{\circledR}$ system, Sci. Rep., 7, 44668. DOI: 10.1038/srep44668.

[6] Brazhnik K., Sokolova Z., Baryshnikova M., Bilan R., Efimov A., Nabiev I., Sukhanova A. (2015) Quantum dot-based lab-on-a-bead system for multiplexed detection of free and total prostate-specific antigens in clinical human serum samples, Nonomedicine: NBM, 11, 1065-1075. DOI: 10.1016/j.nano.2015.03.003.

[7] Sukhorukov G.B., Volodkin D.V., Günther A.M., Petrov A.I., Shenoy D.B., Möhwald H. (2004) Porous calcium carbonate microparticles as templates for encapsulation of bioactive compounds, J. Mater. Chem., 14, 2073-2081. DOI: 10.1039/B402617A.

[8] Bilan R.S., Krivenkov V.A., Berestovoy M.A., Efimov A.E., Agapov I.I., Samokhvalov P.S., Nabiev I., Sukhanova A. (2017) Engineering of optically encoded microbeads with FRET-free spatially separated quantum dot layers for multiplexed assays, ChemPhysChem, 18, 970-979. DOI: 10.1002/cphc.201601274. 